Y. Sun

Nagoya Math. J.

Vol. 173 (2004), 139-151

\title{
ON THE EXISTENCE AND BOUNDEDNESS OF SQUARE FUNCTION OPERATORS ON CAMPANATO SPACES
}

\author{
YONGZHONG SUN
}

\begin{abstract}
Let $g(f)$ be a Littlewood-Paley square function of $f$, which belongs to Campanato spaces $L^{p, \alpha}\left(1<p<\infty,-\frac{n}{p} \leq \alpha<1\right)$. We prove that if $g(f)\left(x_{0}\right)$ exists (i.e. $\left.g(f)\left(x_{0}\right)<\infty\right)$ for a single point $x_{0} \in R^{n}$, then $g(f)(x)$ exists almost everywhere in $R^{n}$ and $\|g(f)\|_{L^{p, \alpha}} \leq C\|f\|_{L^{p, \alpha}}$. Thus we give an improvement of some earlier results such as in [8], where it is always needed to assume $g(f)(x)$ exists in a set of positive measure in order to get the a.e. existence and boundedness of $g(f)(x)$.
\end{abstract}

\section{$\S 1$. Introduction}

In this note we study the existence and boundedness property of square function operators on Campanato spaces. First we introduce the definition of square functions, which are the generalization of classical ones. See [4].

Definition 1.1. $\psi \in C^{1}\left(R^{n}\right)$ is called a Littlewood-Paley function, if there is a positive constant $c$ such that:

(i) $\psi \in L^{1}$ and $\int_{R^{n}} \psi(x) d x=0$;

(ii) $|\psi(x)| \leq c(1+|x|)^{-n-1}$;

(iii) $|\nabla \psi(x)| \leq c(1+|x|)^{-n-2}$.

For a given Littlewood-Paley function, one may define three kinds of square functions.

Definition 1.2. Let $\psi$ be a Littlewood-Paley function and $f \in$ $L_{l o c}\left(R^{n}\right)$, define

Received January 21, 2002.

2000 Mathematics Subject Classification: Primary 42B25, 42B30. 
1. Radial square function.

$$
g_{\psi}(f)(x)=\left(\int_{0}^{\infty}\left|\psi_{t} * f(x)\right|^{2} \frac{d t}{t}\right)^{\frac{1}{2}}
$$

2 Nontangential square function.

$$
s_{\psi}(f)(x)=\left(\int_{|x-y|<t}\left|\psi_{t} * f(y)\right|^{2} \frac{d t d y}{t^{n+1}}\right)^{\frac{1}{2}}
$$

3. Tangential square function.

$$
g_{\lambda, \psi}^{*}(f)(x)=\left(\int_{0}^{\infty} \int_{R^{n}}\left|\psi_{t} * f(x-y)\right|^{2}\left(1+\frac{|y|}{t}\right)^{-\lambda n} \frac{d t d y}{t^{n+1}}\right)^{\frac{1}{2}}
$$

where $\lambda>0$ is a real number, $\psi_{t}(x)=\frac{1}{t^{n}} \psi\left(\frac{x}{t}\right)$.

To be convenient, we omit the subscript $\psi$ and use $g(f), s(f), g_{\lambda}^{*}(f)$ to denote three square functions respectively. Next we introduce the concept of Campanato spaces, which are the generalization of $B M O$ and Lipschitz spaces.

Definition 1.3. ([1]) Suppose $f$ is a locally integrable function and $1 \leq p<\infty,-\frac{n}{p} \leq \alpha<1$. Define

$$
[f]_{L^{p, \alpha}}=\sup _{B}\left(|B|^{-1-\frac{p \alpha}{n}} \int_{B}\left|f(x)-f_{B}\right|^{p} d x\right)^{\frac{1}{p}} .
$$

Campanato space $L^{p, \alpha}=\left\{f \in L_{L o c}: \quad[f]_{L^{p, \alpha}}<\infty\right\}$.

An useful property of a Campanato function is the following

Lemma 1.1. ([2]) Suppose $f \in L^{p, \alpha}, 1 \leq p<\infty$. If $\beta>0$ and $\alpha<$ $\min \{\beta, 1\}$, then for any ball $B=B_{r}\left(x_{0}\right)$

$$
\int_{R^{n}} \frac{\left|f(y)-f_{B}\right|}{r^{n+\beta}+\left|x_{0}-y\right|^{n+\beta}} d y \leq C r^{\alpha-\beta}[f]_{L^{p, \alpha}}
$$

where $C$ is a constant depending only on $n, \alpha$ and $\beta$. 
In [6] the author studied the behavior of classical square functions acting on $L^{\infty}$ and $B M O$ space. He pointed out that the value of square function of an $L^{\infty}$ function may be infinite everywhere in $R^{n}$. Under the assumption of the existence (finiteness) on a set of positive measure, he got the a.e. finiteness and $B M O$-boundedness of square functions. Soon after, such kind of results was generalized to other kind of square functions such as Lusin's area functions, Stein's $g_{\lambda}^{*}$ functions and so on. See [3], for example. Later, in [7] the authors improved aforementioned results in the sense that in order to guarantee the a.e. existence and $B M O$-boundedness of square function, only the assumption of one point finiteness is enough. Similar results also hold for Lipschitz function spaces. See [5]. Since the generalization of $B M O$ and Lipschitz space is Campanato space, a natural question is that if there are similar answers for the square function operators acting on Campanato spaces. Recently, K.Yabuta and others obtained some satisfactory results, see [8] and the references therein. But their results still have a not so satisfied point, that is, in some cases in order to get the a.e. finiteness it is always needed to assume the finiteness of square functions on a positive measure set (such as the first occurrence in [6]). The main results in this note point out that the one point existence of square function is enough. More precisely, we have

TheOrem 1. Suppose $f \in L^{p, \alpha}\left(1<p<\infty,-\frac{n}{p} \leq \alpha<1\right)$. If $g(f)\left(x_{0}\right)<+\infty$ for a single point $x_{0} \in R^{n}$, then $g(f)(x)$ exists almost everywhere in $R^{n}$ and there is a constant $C$ depending on $\psi, n, p, \alpha$ such that

$$
\|g(f)\|_{L^{p, \alpha}} \leq C\|f\|_{L^{p, \alpha}}
$$

Theorem 2. Suppose $f \in L^{p, \alpha}\left(1<p<\infty,-\frac{n}{p} \leq \alpha<1\right)$. If there is a point $x_{0} \in R^{n}$ such that $s(f)\left(x_{0}\right)<+\infty$, then $s(f)(x)<+\infty$ almost everywhere in $R^{n}$ and there is a constant $C$ depending only on $\psi, n, p, \alpha$ such that

$$
\|s(f)\|_{L^{p, \alpha}} \leq C\|f\|_{L^{p, \alpha}} .
$$

Theorem 3. Suppose $f \in L^{p, \alpha}\left(1<p<\infty,-\frac{n}{p} \leq \alpha<1\right), \lambda>3+\frac{2}{n}$. If there is a point $x_{0} \in R^{n}$ such that $g_{\lambda}^{*}(f)\left(x_{0}\right)<+\infty$, then $g_{\lambda}^{*}(f)(x)$ exists almost everywhere in $R^{n}$ and there is a constant $C$ depending only on $\psi, n, p, \lambda, \alpha$ such that

$$
\left\|g_{\lambda}^{*}(f)\right\|_{L^{p, \alpha}} \leq C\|f\|_{L^{p, \alpha}} .
$$


Obviously, our results improve and generalize some earlier results, especially those in [7] and [8].

Finally, We need to point out that though our results are new and the approach is different from others, many of our idea come from $[6,7]$ and $[8]$.

\section{§2. Proof of Theorem 1}

We first deal with radial square function, which is relatively simple.

Let $B=B_{r}\left(x_{0}\right)$ be any ball with centre $x_{0}$. As usual, we decompose $f$ in the following way,

$$
\begin{aligned}
f(x) & =f_{4 B}+\left(f(x)-f_{4 B}\right) \chi_{4 B}(x)+\left(f(x)-f_{4 B}\right) \chi_{\left(R^{n} \backslash 4 B\right)}(x) \\
& =f_{1}+f_{2}(x)+f_{3}(x) .
\end{aligned}
$$

Define

$$
\begin{gathered}
g_{0}(f)(x)=\left(\int_{0}^{r}\left|\psi_{t} * f(x)\right|^{2} \frac{d t}{t}\right)^{\frac{1}{2}} \\
g_{\infty}(f)(x)=\left(\int_{r}^{\infty}\left|\psi_{t} * f(x)\right|^{2} \frac{d t}{t}\right)^{\frac{1}{2}} .
\end{gathered}
$$

Obviously,

$$
g_{0}(f)(x), g_{\infty}(f)(x) \leq g(f)(x) \leq g_{0} f(x)+g_{\infty} f(x)
$$

By property (i) of $\psi$,

$$
g\left(f_{1}\right)=g_{0}\left(f_{1}\right)=g_{\infty}\left(f_{1}\right) \equiv 0
$$

Since $g\left(f_{2}\right)(x) \in L^{p}, g\left(f_{2}\right)(x)$ exists a.e. in $R^{n}$ and

$$
\left\|g\left(f_{2}\right)\right\|_{L^{p}(B)} \leq C\left\|f_{2}\right\|_{L^{p}} \leq C|B|^{\frac{1}{p}+\frac{\alpha}{n}}[f]_{L^{p, \alpha}} ;
$$

In fact we have the following pointwise estimate for $g_{\infty}\left(f_{2}\right)$ :

Lemma 2.1. For any $x \in R^{n}$,

$$
g_{\infty}\left(f_{2}\right)(x) \leq C|B|^{\frac{\alpha}{n}}[f]_{L^{p, \alpha}} .
$$


Proof. By condition (ii) of $\psi$,

$$
\begin{aligned}
g_{\infty}\left(f_{2}\right)(x) & =\left(\int_{r}^{\infty}\left|\int_{R^{n}} \psi_{t}(x-y) f_{2}(y) d y\right|^{2} \frac{d t}{t}\right)^{\frac{1}{2}} \\
& \leq\left(\int_{r}^{\infty}\left(\int_{4 B}\left|\psi_{t}(x-y)\right|\left|f(y)-f_{4 B}\right| d y\right)^{2} \frac{d t}{t}\right)^{\frac{1}{2}} \\
& \leq C\left(\int_{r}^{\infty}\left|\int_{4 B} t^{-n}\left(1+\frac{|x-y|}{t}\right)^{-n-1}\right| f(y)-f_{4 B}|d y|^{2} \frac{d t}{t}\right)^{\frac{1}{2}} \\
& \leq C\left(\int_{4 B}\left|f(y)-f_{4 B}\right| d y\right)\left(\int_{r}^{+\infty} t^{-2 n-1} d t\right)^{\frac{1}{2}} \\
& \leq C r^{-n}\left|f(y)-f_{4 B}\right| d y \leq C|B|^{\frac{\alpha}{n}}[f]_{L^{p, \alpha}} .
\end{aligned}
$$

We need the following two lemmas.

Lemma 2.2. For any $x \in B, g_{0}\left(f_{3}\right)(x)<\infty$ and

$$
g_{0}\left(f_{3}\right)(x) \leq C|B|^{\frac{\alpha}{n}}[f]_{L^{p, \alpha}} .
$$

Proof. Note that if $t<r, x \in B, y \notin 4 B$, then $(t+|x-y|) \sim\left(r+\left|x_{0}-y\right|\right)$.

$$
\begin{aligned}
g_{0}\left(f_{3}\right)(x) & =\left(\int_{0}^{r}\left|\int_{R^{n}} \psi_{t}(x-y) f_{3}(y) d y\right|^{2} \frac{d t}{t}\right)^{\frac{1}{2}} \\
& \leq C\left(\int_{0}^{r}\left|\int_{R^{n}} \frac{1}{t^{n}} \frac{t^{n+1}}{t^{n+1}+|x-y|^{n+1}}\right| f_{3}(y)|d y|^{2} \frac{d t}{t}\right)^{\frac{1}{2}} \\
& \leq C\left(\left.\int_{0}^{r} \int_{R^{n} \backslash 4 B} \frac{\left|f(y)-f_{4 B}\right|}{r^{n+1}+|x-y|^{n+1}} d y\right|^{2} t d t\right)^{\frac{1}{2}} \\
& \leq C r^{\alpha-1}[f]_{L^{p, \alpha}}\left(\int_{0}^{r} t d t\right)^{\frac{1}{2}} \\
& \leq C r^{\alpha}[f]_{L^{p, \alpha}}=C|B|^{\frac{\alpha}{n}}[f]_{L^{p, \alpha}}
\end{aligned}
$$


The next to the last inequality comes from Lemma 1.1.

The following is a key lemma.

Lemma 2.3. For any $x \in B, g_{\infty}\left(f_{3}\right)(x)<\infty$ and

$$
\left|g_{\infty}\left(f_{3}\right)(x)-g_{\infty}(f)\left(x_{0}\right)\right| \leq C|B|^{\frac{\alpha}{n}}[f]_{L^{p, \alpha}} .
$$

Note that by assumption, $g_{\infty}(f)\left(x_{0}\right) \leq g(f)\left(x_{0}\right)<\infty$.

Proof. By property (i) of $\psi$,

$$
\begin{aligned}
& \left|g_{\infty}\left(f_{3}\right)(x)-g_{\infty}(f)\left(x_{0}\right)\right| \\
= & \mid\left(\int_{r}^{+\infty}\left|\int_{R^{n}} \psi_{t}(x-y) f_{3}(y) d y\right|^{2} \frac{d t}{t}\right)^{\frac{1}{2}} \\
& -\left(\int_{r}^{+\infty}\left|\int_{R^{n}} \psi_{t}\left(x_{0}-y\right) f(y) d y\right|^{2} \frac{d t}{t}\right)^{\frac{1}{2}} \mid \\
\leq & \left(\int_{r}^{+\infty}\left|\int_{R^{n}}\left[\psi_{t}(x-y) f_{3}(y)-\psi_{t}\left(x_{0}-y\right) f(y)\right] d y\right|^{2} \frac{d t}{t}\right)^{\frac{1}{2}} \\
\leq & \left(\int_{r}^{+\infty}\left|\int_{R^{n}}\left[\psi_{t}(x-y)-\psi_{t}\left(x_{0}-y\right)\right] f_{3}(y) d y\right|^{2} \frac{d t}{t}\right)^{\frac{1}{2}}+g_{\infty}\left(f_{2}\right)\left(x_{0}\right) \\
= & I(x)+g_{\infty}\left(f_{2}\right)\left(x_{0}\right) ; \\
I(x) \leq & \int_{R^{n} \backslash 4 B}\left|f(y)-f_{4 B}\right|\left(\int_{r}^{+\infty}\left|\psi_{t}(x-y)-\psi_{t}\left(x_{0}-y\right)\right|^{2} \frac{d t}{t}\right)^{\frac{1}{2}} d y ;
\end{aligned}
$$

For $\forall x \in B, y \notin 4 B,\left|\psi(x-y)-\psi\left(x_{0}-y\right)\right| \leq C\left|x-x_{0}\right|\left(1+\left|x_{0}-y\right|\right)^{-(n+2)}$.

$$
\begin{aligned}
& \left(\int_{r}^{+\infty}\left|\psi_{t}(x-y)-\psi_{t}\left(x_{0}-y\right)\right|^{2} \frac{d t}{t}\right)^{\frac{1}{2}} \\
\leq & C\left(\int_{r}^{+\infty} \frac{1}{t^{2 n}}\left[\frac{t^{-1}\left|x-x_{0}\right|}{\left(1+t^{-1}\left|x_{0}-y\right|\right)^{n+2}}\right]^{2} \frac{d t}{t}\right)^{\frac{1}{2}}
\end{aligned}
$$




$$
\begin{aligned}
& \leq C r\left(\int_{0}^{+\infty}\left(1+\frac{\left|x_{0}-y\right|}{t}\right)^{-2(n+2)} \frac{d t}{t^{2 n+3}}\right)^{\frac{1}{2}} \\
& =C \frac{r}{\left|x_{0}-y\right|^{n+1}}\left(\int_{0}^{+\infty} \frac{s}{(1+s)^{2(n+2)}} d s\right)^{\frac{1}{2}} \\
& =C \frac{r}{\left|x_{0}-y\right|^{n+1}}
\end{aligned}
$$

Hence by Lemma 1.1,

$$
I(x) \leq C r \int_{R^{n} \backslash 4 B} \frac{\left|f(y)-f_{4 B}\right|}{\left|x_{0}-y\right|^{n+1}} d y \leq C r^{\alpha}[f]_{L^{p, \alpha}} .
$$

Finally, using Lemma 2.1,

$$
\left|g_{\infty}\left(f_{3}\right)(x)-g_{\infty}(f)\left(x_{0}\right)\right| \leq I(x)+g_{\infty}\left(f_{2}\right)\left(x_{0}\right) \leq C|B|^{\frac{\alpha}{n}}[f]_{L^{p, \alpha}} .
$$

Remark 2.1. In fact, in the estimate of the term $I$ we proved

$$
\left(\int_{0}^{+\infty}\left|\psi_{t}(x-y)-\psi_{t}(x)\right|^{2} \frac{d t}{t}\right)^{\frac{1}{2}} \leq C|y||x|^{-(n+1)} .
$$

We continue to prove the theorem. By Lemmas 2.2 and 2.3, we know $g\left(f_{3}\right)(x)$ is finite everywhere in $B$. By the a.e. existence of $g\left(f_{2}\right)(x)$ we know $g(f)(x)$ is finite a.e. in $B$. By the arbitrarity of the radius of $B=B_{r}\left(x_{0}\right)$, we get the a.e. existence of $g(f)(x)$ in $R^{n}$. Next we prove the boundedness of $g(f)$.

Let $E=\left\{x \in R^{n} \mid g(f)(x)<\infty\right\}$. Obviously, one only needs to prove that for any ball $B^{*}=B_{\rho}\left(x^{*}\right)$ with centre $x^{*} \in E$,

$$
\left(\int_{B^{*}}\left|g(f)(x)-(g(f))_{B^{*}}\right|^{p} d x\right)^{\frac{1}{p}} \leq C\left|B^{*}\right|^{\frac{1}{p}+\frac{\alpha}{n}}[f]_{L^{p, \alpha}} .
$$

In fact, let

$$
\begin{aligned}
f(x) & =f_{4 B^{*}}+\left(f(x)-f_{4 B^{*}}\right) \chi_{4 B^{*}}(x)+\left(f(x)-f_{4 B^{*}}\right) \chi_{\left(R^{n} \backslash 4 B^{*}\right)}(x) \\
& =h_{1}+h_{2}(x)+h_{3}(x)
\end{aligned}
$$

and denote

$$
g_{0}(f)(x)=\left(\int_{0}^{\rho}\left|\psi_{t} * f(x)\right|^{2} \frac{d t}{t}\right)^{\frac{1}{2}}
$$




$$
g_{\infty}(f)(x)=\left(\int_{\rho}^{\infty}\left|\psi_{t} * f(x)\right|^{2} \frac{d t}{t}\right)^{\frac{1}{2}} .
$$

Repeating the argument of proving (2.1), (2.2), (2.4) and (2.5), we have

$$
\begin{gathered}
g\left(h_{1}\right)=g_{0}\left(h_{1}\right)=g_{\infty}\left(h_{1}\right) \equiv 0 \\
\left\|g\left(h_{2}\right)\right\|_{L^{p}\left(B^{*}\right)} \leq C\left\|h_{2}\right\|_{L^{p}} \leq C\left|B^{*}\right|^{\frac{1}{p}+\frac{\alpha}{n}}[f]_{L^{p, \alpha}} \\
\sup _{x \in B^{*}} g_{0}\left(h_{3}\right)(x) \leq C\left|B^{*}\right|^{\frac{\alpha}{n}}[f]_{L^{p, \alpha}} ; \\
\sup _{x \in B^{*}}\left|g_{\infty}\left(h_{3}\right)(x)-g_{\infty}(f)\left(x^{*}\right)\right| \leq C\left|B^{*}\right|^{\frac{\alpha}{n}}[f]_{L^{p, \alpha}} .
\end{gathered}
$$

Hence

$$
\begin{aligned}
& \left(\int_{B^{*}}\left|g(f)(x)-[g(f)]_{B^{*}}\right|^{p} d x\right)^{\frac{1}{p}} \\
\leq & 2\left(\int_{B^{*}}\left|g(f)(x)-g_{\infty}(f)\left(x^{*}\right)\right|^{p} d x\right)^{\frac{1}{p}} \\
\leq & 2\left(\int_{B^{*}}\left|g(f)(x)-g\left(h_{3}\right)(x)\right|^{p} d x\right)^{\frac{1}{p}} \\
\leq & 2\left(\int_{B^{*}}\left|g\left(h_{2}\right)(x)\right|^{p}\right)^{\frac{1}{p}}+2\left(\int_{B^{*}}\left|g\left(h_{3}\right)(x)-g_{\infty}(f)\left(x^{*}\right)\right|^{p} d x\right)^{\frac{1}{p}} \\
& \left.+\left.2\left(\int_{B^{*}}\left|g_{\infty}\left(h_{3}\right)(x)-g_{\infty}(f)\left(x^{*}\right)\right|^{p} d x\right)^{\frac{1}{p}} g_{\infty}\left(h_{3}\right)(x)\right|^{p} d x\right)^{\frac{1}{p}} \\
\leq & 2\left\|g\left(h_{2}\right)\right\|_{L^{p}\left(B^{*}\right)}+2\left(\int_{B^{*}}\left|g_{0}\left(h_{3}\right)(x)\right|^{p} d x\right)^{\frac{1}{p}} \\
& +2\left|B^{*}\right|^{\frac{1}{p}} \sup _{x \in B^{*}}\left|g_{\infty}\left(h_{3}\right)(x)-g_{\infty}(f)\left(x^{*}\right)\right| \\
\leq & C\left|B^{*}\right| \frac{1}{p}+\frac{\alpha}{n}[f]_{L^{p, \alpha} .}
\end{aligned}
$$

\section{$\S 3$. Proof of Theorems 2, 3}

We only give the proof of Theorem 3 . The proof of Theorem 2 is similar and we leave it to the interesting reader. 
As in the proof of Theorem 1, we only need to prove the following three lemmas concerning on $g_{\lambda}^{*}$. Use the same notation as the last section, define

$$
\begin{aligned}
g_{\lambda, 0}^{*}(f)(x) & =\left(\int_{0}^{r} \int_{R^{n}}\left|\psi_{t} * f(x-u)\right|^{2} d \mu\right)^{\frac{1}{2}} ; \\
g_{\lambda, \infty}^{*}(f)(x) & =\left(\int_{r}^{\infty} \int_{R^{n}}\left|\psi_{t} * f(x-u)\right|^{2} d \mu\right)^{\frac{1}{2}} ;
\end{aligned}
$$

where $d \mu=\left(1+\frac{|u|}{t}\right)^{-\lambda n} \frac{d u d t}{t^{n+1}}$.

Lemma 3.1. For any $x \in R^{n}, g_{\lambda, \infty}^{*}\left(f_{2}\right)(x)<\infty$ and

$$
g_{\lambda, \infty}^{*}\left(f_{2}\right)(x) \leq C|B|^{\frac{\alpha}{n}}[f]_{L^{p, \alpha}}
$$

where the constant $C$ depends only on $\psi, n, p, \alpha$ and $\lambda$.

Proof. By noting condition (ii) of $\psi$,

$$
\begin{aligned}
g_{\lambda, \infty}^{*}\left(f_{2}\right)(x) & =\left(\int_{r}^{\infty} \int_{R^{n}}\left|\int_{R^{n}} \psi_{t}(x-y-u) f_{2}(y) d y\right|^{2} d \mu\right)^{\frac{1}{2}} \\
& \leq\left(\int_{r}^{\infty} \int_{R^{n}}\left[\int_{4 B}\left|\psi_{t}(x-u-y)\right|\left|f(y)-f_{4 B}\right| d y\right]^{2} d \mu\right)^{\frac{1}{2}} \\
& \leq C\left(\int_{r}^{\infty} \int_{R^{n}}\left[\int_{4 B} \frac{\left|f(y)-f_{4 B}\right| d y}{t^{n}\left(1+t^{-1}|x-y-u|\right)^{n+1}}\right]^{2} d \mu\right)^{\frac{1}{2}} \\
& \leq C \int_{4 B}\left|f(y)-f_{4 B}\right| d y\left(\int_{r}^{+\infty} \int_{R^{n}}\left(\frac{t}{t+|u|}\right)^{\lambda n} \frac{d u d t}{t^{3 n+1}}\right)^{\frac{1}{2}} \\
& \leq C \int_{4 B}\left|f(y)-f_{4 B}\right| d y\left(\int_{r}^{+\infty} \int_{R^{n}}\left(\frac{1}{1+|v|}\right)^{\lambda n} \frac{d v d t}{t^{2 n+1}}\right)^{\frac{1}{2}} \\
& \leq C \frac{1}{r^{n}} \int_{4 B}\left|f(y)-f_{4 B}\right| d y \leq C|B|^{\frac{\alpha}{n}}[f]_{L^{p, \alpha}}
\end{aligned}
$$

The following lemma is essentially contained in Lemma 4 in [7].

LEMMA 3.2. If $\lambda>3+\frac{2}{n}$, then for any $x \in B, g_{\lambda, 0}^{*}\left(f_{3}\right)(x)$ exists and

$$
g_{\lambda, 0}^{*}\left(f_{3}\right)(x) \leq C|B|^{\frac{\alpha}{n}}[f]_{L^{p, \alpha}} .
$$


Proof. By condition (ii) of $\psi$ and $(1+|x|) \leq(1+|x-y|)(1+|y|), \forall x, y \in$ $R^{n}$

$$
\begin{aligned}
& g_{\lambda, 0}^{*}\left(f_{3}\right)(x) \\
= & \left(\int_{0}^{r} \int_{R^{n}}\left|\int_{R^{n}} \psi_{t}(x-y-u) f_{3}(y) d y\right|^{2} d \mu\right)^{\frac{1}{2}} \\
\leq & C\left[\int_{0}^{r} \int_{R^{n}}\left(\int_{R^{n} \backslash 4 B} \frac{t\left|f(y)-f_{4 B}\right|}{(t+|x-u-y|)^{n+1}} d y\right)^{2}\left(\frac{t}{t+|u|}\right)^{\lambda n} \frac{d u d t}{t^{n+1}}\right]^{\frac{1}{2}} \\
\leq & C \int_{R^{n} \backslash 4 B}\left|f(y)-f_{4 B}\right|\left(\int_{0}^{r} \int_{R^{n}} \frac{t\left(1+\frac{|u|}{t}\right)^{-\lambda n+2 n+2}}{(t+|x-y|)^{2(n+1)}} \frac{d u d t}{t^{n}}\right)^{\frac{1}{2}} d y
\end{aligned}
$$

Noting that for $t \in(0, r), x \in B, y \notin 4 B$, one has $t+|x-y| \sim r+\left|x_{0}-y\right|$ and $\lambda>3+\frac{2}{n}$,

$$
\begin{aligned}
g_{\lambda, 0}^{*}\left(f_{3}\right)(x) & \leq C r \int_{R^{n} \backslash 4 B} \frac{\left|f(y)-f_{4 B}\right|}{\left(r+\left|x_{0}-y\right|\right)^{n+1}} d y \\
& \leq C r^{\alpha}[f]_{L^{p, \alpha}}=C|B|^{\frac{\alpha}{n}}[f]_{L^{p, \alpha}} ;
\end{aligned}
$$

The last inequality comes from Lemma 1.1.

LEMмA 3.3. If $\lambda>3+\frac{2}{n}$, then for any $x \in B, g_{\lambda, \infty}^{*}\left(f_{3}\right)(x)<\infty$ and

$$
\left|g_{\lambda, \infty}^{*}\left(f_{3}\right)(x)-g_{\lambda, \infty}^{*}(f)\left(x_{0}\right)\right| \leq C|B|^{\frac{\alpha}{n}}[f]_{L^{p, \alpha}} .
$$

Proof. By Lemma 3.1 and using the same argument as in the proof of Lemma 2.3, one only needs to prove

$$
\begin{aligned}
I(x) & =\left(\int_{R_{+}^{n+1}}\left|\int_{R^{n}}\left[\psi_{t}(x-u-y)-\psi_{t}\left(x_{0}-u-y\right)\right] f_{3}(y) d y\right|^{2} d \mu\right)^{\frac{1}{2}} \\
& \leq C r \int_{R^{n} \backslash 4 B} \frac{\left|f(y)-f_{4 B}\right| d y}{\left|x_{0}-y\right|^{n+1}} ;
\end{aligned}
$$

By the Minkowski inequality, we only need to estimate the following term $I I(x)$. By using condition (ii) of $\psi$,

$$
I I(x)=\left(\int_{R_{+}^{n+1}}\left|\psi_{t}(x-u-y)-\psi_{t}\left(x_{0}-u-y\right)\right|^{2} d \mu\right)^{\frac{1}{2}}
$$




$$
\begin{aligned}
& =\left(\int_{R_{+}^{n+1}}\left|\int_{0}^{1}\left(x-x_{0}\right) \cdot \nabla \psi_{t}\left(x_{0}-u-y+\theta\left(x-x_{0}\right)\right) d \theta\right|^{2} d \mu\right)^{\frac{1}{2}} \\
& \leq C \int_{0}^{1}\left(\int_{R_{+}^{n+1}} \frac{\left|x-x_{0}\right|^{2}}{t^{2 n+2}\left(1+t^{-1}\left|x_{0}-u-y+\theta\left(x-x_{0}\right)\right|\right)^{2(n+2)}} d \mu\right)^{\frac{1}{2}} d \theta \\
& \leq C r \int_{0}^{1}\left(\int_{R_{+}^{n+1}} \frac{(1+|v|)^{-\lambda n} d v d t}{t^{2 n+3}\left(1+\left|\frac{x_{0}-y+\theta\left(x-x_{0}\right)}{t}-v\right|\right)^{2(n+2)}}\right)^{\frac{1}{2}} d \theta \\
& =C r \int_{0}^{1}|z|^{-n-1}\left(\int_{R_{+}^{n+1}} \frac{(1+|v|)^{-\lambda n} d v d s}{s^{2 n+3}\left(1+\left|s^{-1} z^{\prime}-v\right|\right)^{2(n+2)}}\right)^{\frac{1}{2}} d \theta \\
& =C r \int_{0}^{1}|z|^{-n-1}\left(\int_{0}^{+\infty} \frac{h_{z^{\prime}}(s) d s}{s^{2 n+3}}\right)^{\frac{1}{2}} d \theta
\end{aligned}
$$

where $z=\left|x_{0}-y+\theta\left(x-x_{0}\right)\right|, z^{\prime}=z|z|^{-1}, h_{z^{\prime}}(s)=\int_{R^{n}} \frac{(1+|v|)^{-\lambda n} d v}{\left(1+\left|s^{-1} z^{\prime}-v\right|\right)^{2(n+2)}}$. Next we estimate $h_{z^{\prime}}(s)$.

If $s \geq 1$, then

$$
h_{z^{\prime}}(s) \leq \int_{R^{n}} \frac{d v}{(1+|v|)^{\lambda n}}=C(n, \lambda)
$$

If $s<1$, then

$$
h_{z^{\prime}}(s) \leq \int_{|v|<\frac{1}{2 s}}+\int_{\frac{1}{2 s} \leq|v| \leq \frac{2}{s}}+\int_{|v|>\frac{2}{s}}
$$

Note that if $|v|<\frac{1}{2 s}$, then $\left|s^{-1} z^{\prime}-v\right| \geq \frac{1}{2 s}$,

$$
\int_{|v|<\frac{1}{2 s}} \frac{(1+|v|)^{-\lambda n} d v}{\left(1+\left|s^{-1} z^{\prime}-v\right|\right)^{2(n+2)}} \leq 2^{n} \int_{R^{n}} \frac{s^{2(n+2)}(1+|v|)^{-\lambda n} d v}{(1+2 s)^{2(n+2)}} \leq C s^{2(n+2)}
$$

If $\frac{1}{2 s} \leq|v| \leq \frac{2}{s}$, then $1+|v| \sim \frac{1}{s}$

$$
\begin{aligned}
\int_{\frac{1}{2 s} \leq|v| \leq \frac{2}{s}} & \frac{(1+|v|)^{-\lambda n} d v}{\left(1+\left|s^{-1} z^{\prime}-v\right|\right)^{2(n+2)}} \\
& \leq C \int_{\frac{1}{2 s} \leq|v| \leq \frac{2}{s}} \frac{s^{\lambda n} d v}{\left(1+\left|s^{-1} z^{\prime}-v\right|\right)^{2(n+2)}} \leq C s^{(\lambda-1) n} ;
\end{aligned}
$$




$$
\begin{aligned}
& \text { If }|v|>\frac{2}{s} \text {, then }\left|s^{-1} z^{\prime}-v\right| \geq|v|-\frac{1}{s} \geq \frac{1}{2}|v|, \\
& \qquad \int_{|v|>\frac{2}{s}} \frac{(1+|v|)^{-\lambda n} d v}{\left(1+\left|s^{-1} z^{\prime}-v\right|\right)^{2(n+2)}} \leq C \int_{|v|>\frac{2}{s}}|v|^{-\lambda n-2 n-4} d v \leq C s^{\lambda n+2 n+3} ;
\end{aligned}
$$

Finally by noting $\lambda>3+\frac{2}{n},(\lambda-1) n>2 n+2$,

$$
\int_{0}^{+\infty} \frac{h_{z^{\prime}}(s) d s}{s^{2 n+3}} \leq C
$$

hence

$$
I I(x) \leq C r \int_{0}^{1}|z|^{-n-1} d \theta \leq C r\left|x_{0}-y\right|^{-n-1}
$$

and (3.5) is proved.

Remark 3.1. We need the assumption of $\lambda>3+\frac{2}{n}$, while in [8] only $\lambda>1+\frac{2}{n}$ is assumed.

Acknowledgements. I would like to thank the referee for careful reading and pointing out a mistake in the proof of Lemma 3.3 in the earlier version of this paper.

\section{REFERENCES}

[1] S. Campanato, Proprietà di hölderianità di alcune di funzioni, Ann. Scuola Norm. Sup. Pisa, 17 (1963), 175-188.

[2] F. B. Fabes, R. L. Janson \& U. Neri, Spaces of harmonic functions representable by Poisson integrals of functions in $B M O$ and $\mathcal{L}_{p, \lambda}$, Indiana Univ. Math. J., 25 (1976), 159-170.

[3] D. S. Kurtz, Littlewood-Paley operators on BMO, Proc. Amer. Math. Soc., 99 (1987), 657-666.

[4] A. Torchinsky, Real-variable methods in harmonic analysis, Academic Press, San Diego, Calif., 1986.

[5] Wang Shilin, Boundedness of the Littlewood-Paley g-functions on Lip $\left(R^{n}\right)$ $(0<\alpha<1)$, Ill. J. Math., 33 (1989), 531-541.

[6] Wang Silei, Some properties of g-functions, Science in China, Series A, 10 (1984), 890-899.

[7] Wang Silei \& Chen Jiecheng, Some notes on square function operator, Chinese Annals of Mathematics, Series A, 11 (1990), 630-638.

[8] K. Yabuta, Boundedness of Littlewood-Paley operators, Math. Japonica, 43 (1996), $143-150$. 
Depatment of Mathematics

Nanjing University

Nanjing, Jiangsu

P. R. China

syz411@yahoo.comcn 